\title{
Decreasing Unintended Medication Discrepancies in Medication Reconciliation through Simple Yet Effective Interventions
}

\author{
Hassan Tahir ${ }^{1, *}$, Nagadarshini Ramagiri Vinod ${ }^{1}$, Vistasp Daruwalla ${ }^{2}$, Muhammad Umair Malik ${ }^{1}$, \\ Nikath Zeeshan ${ }^{1}$, Lavanya Vuppu ${ }^{1}$, Thomas Simunich ${ }^{3}$, Medha Joshi ${ }^{1}$ \\ ${ }^{1}$ Temple University, Conemaugh Memorial Hospital, Johnstown, PA, USA \\ ${ }^{2}$ Wyne State University, Detroit Medical Center, Detroit, MI, USA \\ ${ }^{3}$ Statistitician, Conemaugh Memorial Hospital \\ *Corresponding author: hassantahir_01@hotmail.com
}

\begin{abstract}
Background: Medication reconciliation is the process of comparing a patient's medication orders to all of the medications that the patient has been taking. Unfortunately, medication errors are common in our health system accounting for significant proportion of patient harm. The main objective of our study was to explore the effect of self-designed intervention in improving the accuracy of patient current medication list in the outpatient settings. Methods: The study designed entailed collection of data from October 2013 to March 2014. The data collected in October (The pre-intervention) was compared to the post-intervention data in March 2014. Following interventions were performed: Education of residents, nurses and patients, frequent reminders to the residents to perform medication reconciliation, sending notifications to the residents who failed to perform medication reconciliation, reminding the patients get all refills at the time of appointment and reminding the patients to bring pill bottles at each appointment. The pre-intervention data was compared with the post-intervention data for different types of medication errors. Normality of the variables was pre assessed. Non-parametric analysis using Fisher's exact test was performed for comparison of categorical variables. Conclusion: In conclusion, our intervention improved the accuracy of patient current medication list in the electronic records as accurate as possible. For clinics and hospitals, medication reconciliation can enhance delivery of high value cost conscious care to the patients by reducing medication errors.
\end{abstract}

Keywords: medication reconciliation, medication error, patient harm

Cite This Article: Hassan Tahir, Nagadarshini Ramagiri Vinod, Vistasp Daruwalla, Muhammad Umair Malik, Nikath Zeeshan, Lavanya Vuppu, Thomas Simunich, and Medha Joshi, "Decreasing Unintended Medication Discrepancies in Medication Reconciliation through Simple Yet Effective Interventions.” American Journal of Public Health Research, vol. 5, no. 2 (2017): 30-35. doi: 10.12691/ajphr-5-2-1.

\section{Introduction}

Medical errors are often inevitable. Medical reconciliation is one of the processes where medical errors are frequently noted. Thomsen et al in a systematic review accrued evidence from twenty nine studies. The authors reported a median adverse drug event of $14.9 \%$ per 1000 person months. The preventable adverse drug event was $5.6 \%$ per 1000 person months [1]. Medication reconciliation is commonly described as the process of comparing the prescribed patient's medication list to the actual number of medications that the patient has been taking.

Medication reconciliation activities have mainly scrutinized during times of admission, transition or immediately following hospital discharge. Different and independent member of the medical team including nurses, residents, pharmacy and the primary physician usually performs medical reconciliation. This allows identifying current medications in use, discrepancies in medications prescribed and utilized by the patient with prompt correction of the errors. Medical reconciliation involves rigorous checking and patient education to make sure the variances in medications and their unintended usage is monitored as these inaccuracies lead to increased readmissions and adverse drug side effects.

Majority of the patients during their hospitalization are substantially reliant on the medical and ancillary team for addressing their medications. The sudden burden of managing their own medications and recovery at discharge with inadequate supervision and assistance leads to erroneous medication use. Such mismanagement and medication discrepancies are further exaggerated by factors like language barrier, severe illness, patient's comprehension of his medication regimen and familial support in appropriately monitoring the regimen.

The objective of this study was to retrospectively identify the medication discrepancies in discharged patients by review the medication reconciliation. After which a six months of intervention was performed during which included educating physician and patients about the 
importance of medication reconciliation and providing patients with periodic reminders. Post six month intervention the medical records were again reviewed to evaluate for medication discrepancies.

\section{Methods}

\subsection{Study Design and Baseline Characteristics}

A retrospective chart review was performed of 220 consecutive patients visiting the outpatient clinic for the month of October 2013 at the Conemaugh memorial medical center to assess for medication discrepancies. This group was labeled as Pre-intervention group. This study was approved by an institutional review board at Conemaugh memorial medical center. The requirement of individual patient consent was waived based on the retrospective nature of this analysis. As a part of the hospital policy patients are scheduled a follow up appointment 1 month following discharge to evaluate their clinical status.

Study data characteristics like age, sex, primary organ system involved or diagnostic system, type medication errors, failure to add a new or over the counter medication, failure to omit an old medication and inaccuracy in medication dose or frequency were collected. Patients were screened with the application of predetermined inclusion and exclusion criteria.

\subsection{Inclusion Criteria}

- All consecutive patients visiting in the Internal Medicine clinic at the Conemaugh medical center in the month of October 2013(pre-intervention group) and March 2014 (post-intervention group)

Exclusion criteria:

- Patient inability to describe medications

- Patients who visited the clinic for flu shots.

- Patient unreachable on three attempts of phone call.

- Patient who left half way house or changed their primary care.

\subsection{Intervention}

Following steps of intervention were undertaken to improve medication reconciliation during the intervention period from October to March:

1. Education of residents, nurses, physicians and patients about their medication regimen.

a. Residents and nurses were taught the importance and hazards of deficient medication reconciliation during weekly conferences.

b. Residents were instructed to verbally promote and discuss medication regimen with patients.

c. Residents and nurses directed to remind patients to bring pill bottles to every visit and to review patient pill bottles during visit, e.g. appropriateness, interaction with over the counter (OTCs) drugs and herbals, refill status, prescription requirements, etc.

d. Residents encouraged faxing discharge medication list to primary physician. e. Frequent reminders to the residents to perform medication reconciliation.

f. Sending notifications to the residents who failed to perform medication reconciliation.

2. Visual Cueing

a. Reminder stickers placed on resident computers.

b. Reminder posters placed in waiting area and exam rooms.

c. Patient specific reminder to bring pill bottles to every visit also included on appointment cards.

Written notification was sent to residents who failed to perform adequate medication reconciliation as per standard operating procedure during intervention period.

3. Educating patient and providing appropriate supervision through phone call from the clinic nurses or residents prior to outpatient visit:

a. Reminding the patients get all refills at the time of appointment.

b. Reminding the patients to bring pill bottles at each appointment.

\subsection{Post-intervention and Statistical Analysis}

A retrospective chart review was performed of 220 consecutive patients visiting the outpatient clinic for the month of March 2014 at the Conemaugh memorial medical center to assess for medication discrepancies.

Normality of the variables was pre assessed. Nonparametric statistical analysis using 2-sided Fisher's exact test to determine the significance with a $p=.05$ was performed for comparison of categorical variables. All statistical procedures were performed by SPSS 20.0 (IBM SPSS Inc, Chicago, IL).

\section{Results}

A total of 440 patients were reviewed of which 60 were included in the Pre-intervention group and 68 were included in the Post-intervention group after application of the predetermined inclusion and exclusion criteria (Table 1). Pre-intervention group demonstrated a total of 34 females and 26 males with an average age of 52.50 years. The Post-intervention group demonstrated a total of 44 females and 24 males with an average age of 51 years. No statistically significant difference was noted between both the groups (Table 2).

Different primary organ system involved or diagnostic system (Dx category) for the pre and post-intervention group are shown in Table 3.

Table 4 shows the various types of medical errors noted in both the groups. Failure to omit old medications reduced from 27 to 23 patients. Failure to add new medication reduced from 17 to 10 patients. Failure to include OTC drugs was constant at 14 patients in the pre and post-intervention group. Errors in total dose of the medication decreased from 11 to 3 patients. Dosage frequency was 5 and 6 patients in the pre and post-intervention group, respectively. Total number of errors in the pre and post-intervention groups were 75 and 56 patients, respectively, which were statistically significant.

Number of patient charts without any medical errors in the pre-intervention group was 13 and in the 
post-intervention group was 38 that was a statistically significant improvement. Our results suggest an increase in number of charts with no error from 22\% to $56 \%(\mathrm{p}<0.0002)$ and reduction in any type of errors from $61 \%$ to $39 \%(p<0.001)$ after a self-designed intervention.

Table 1
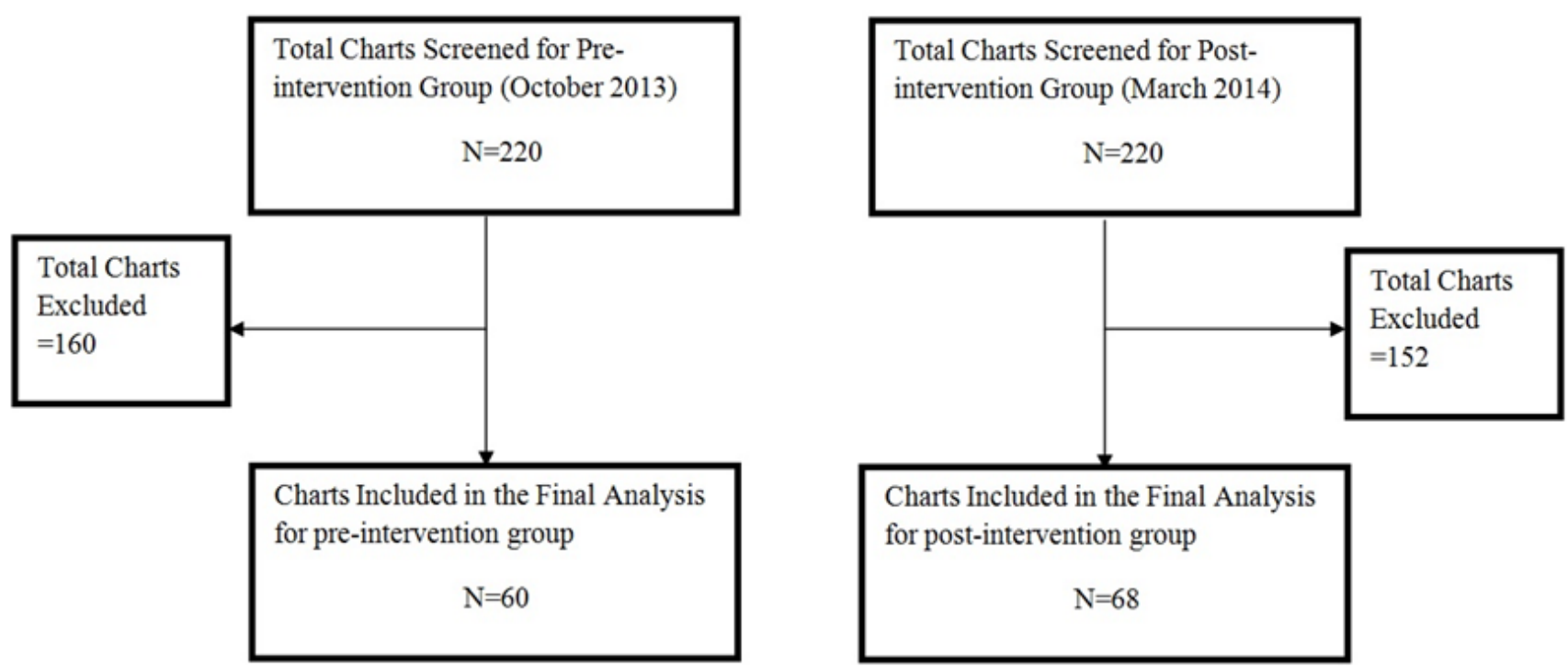

Table 2.

sex

\begin{tabular}{|c|c|c|c|c|c|c|}
\hline \multicolumn{3}{|c|}{ study time period } & Frequency & Percent & Valid Percent & $\begin{array}{l}\text { Cumulative } \\
\text { Percent }\end{array}$ \\
\hline \multirow[t]{3}{*}{ pre } & \multirow[t]{3}{*}{ Valid } & female & 34 & 56.7 & 56.7 & 56.7 \\
\hline & & male & 26 & 43.3 & 43.3 & 100.0 \\
\hline & & Total & 60 & 100.0 & 100.0 & \\
\hline \multirow[t]{3}{*}{ post } & \multirow[t]{3}{*}{ Valid } & female & 44 & 64.7 & 64.7 & 64.7 \\
\hline & & male & 24 & 35.3 & 35.3 & 100.0 \\
\hline & & Total & 68 & 100.0 & 100.0 & \\
\hline
\end{tabular}

Table 3.

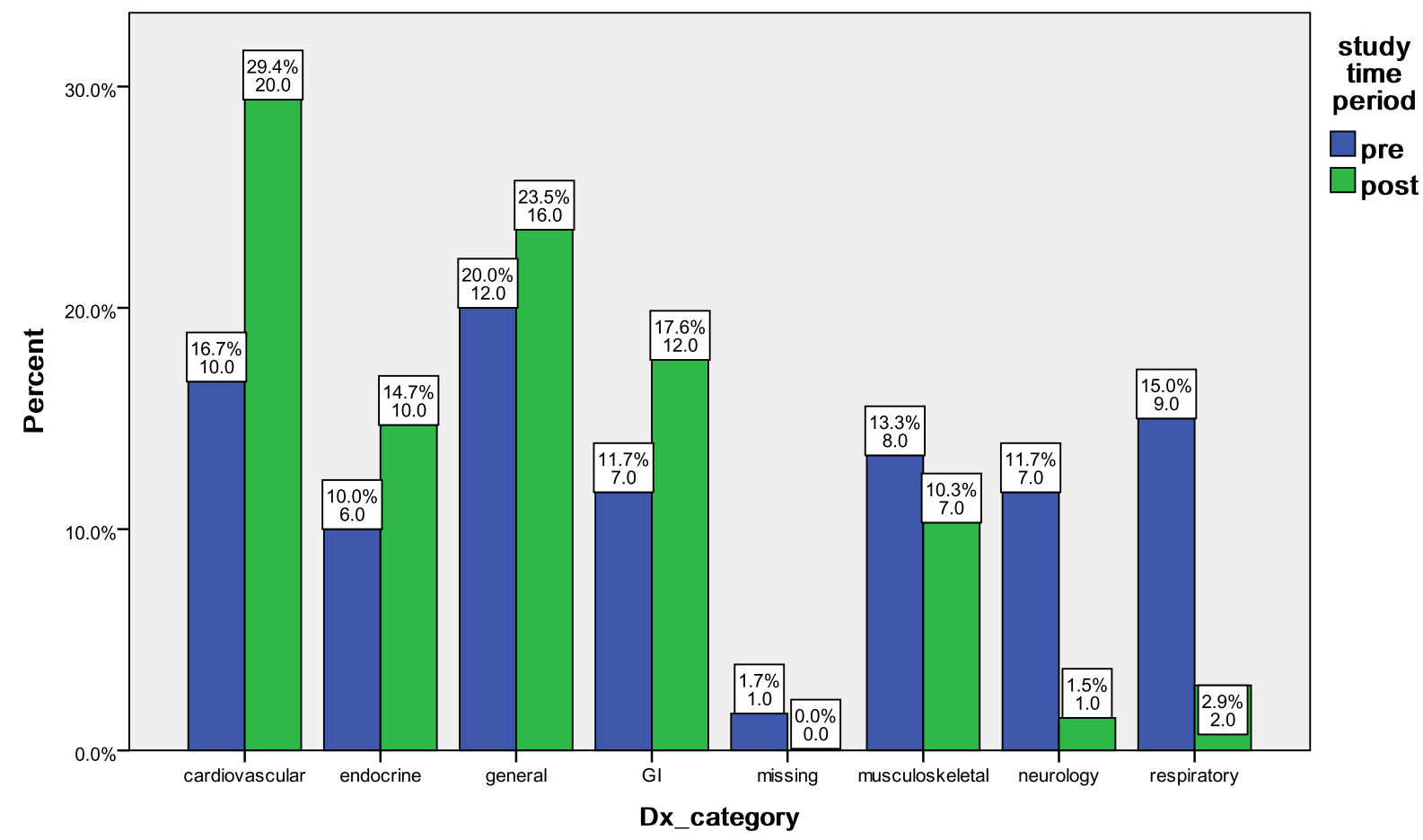


Table 4.

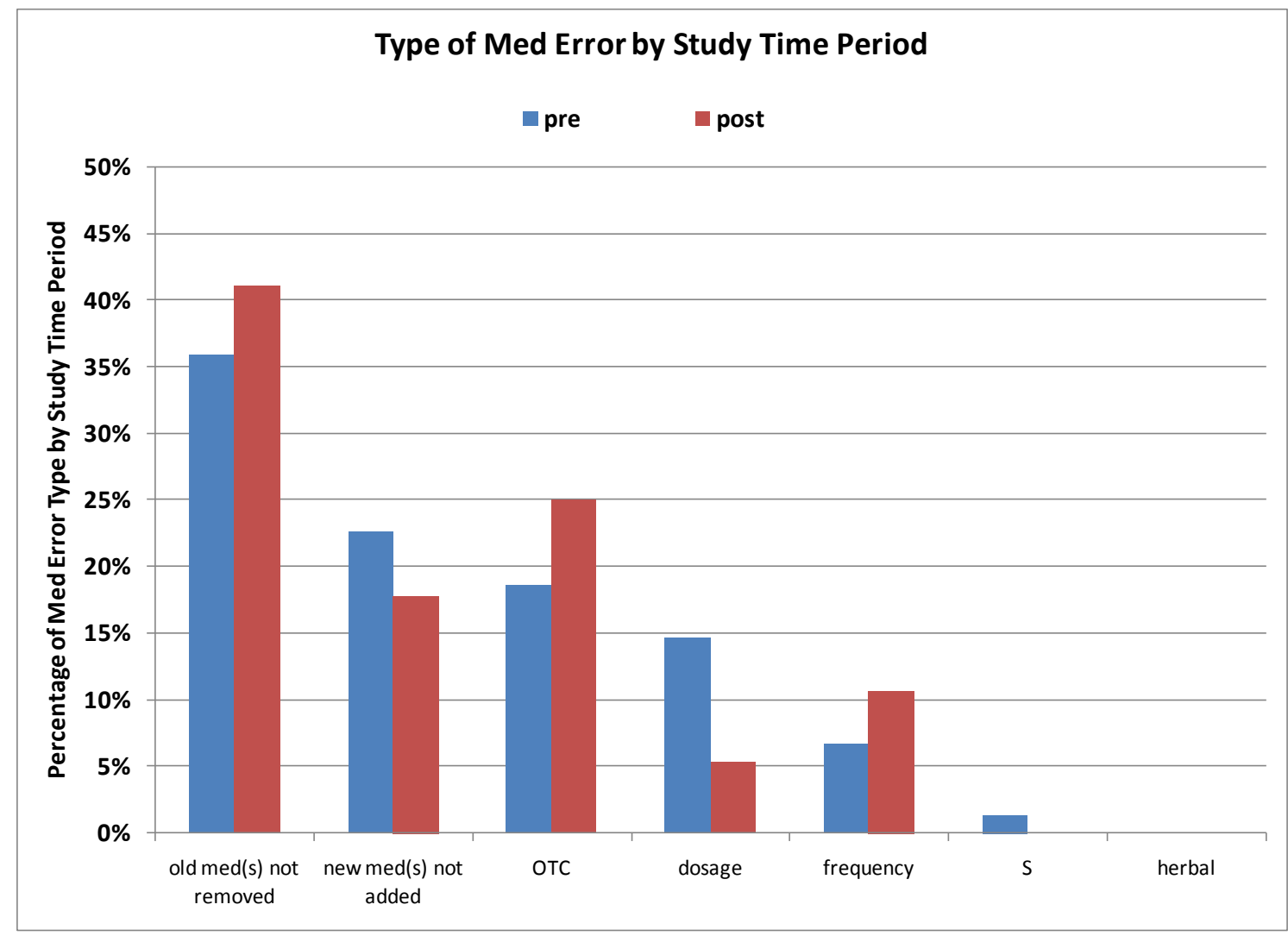

Table 5.

Number of Charts with No Medication Errors

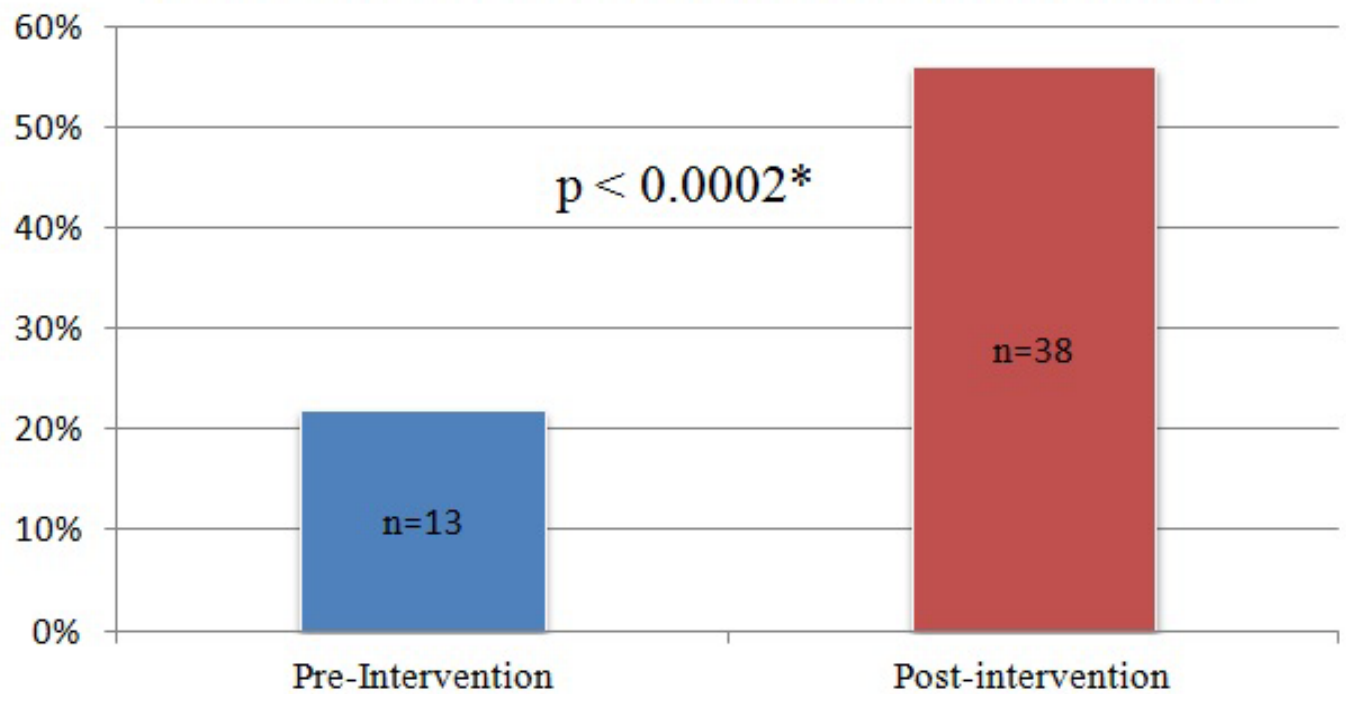

\section{Discussion}

Medical reconciliation is the process of reviewing and creating the most accurate list of patient medications at each transition of care. The purpose of medication reconciliation is to decrease medical error and improve patient safety [2]. Medical reconciliation is a multistep process that involves reviewing and verifying patients list of medications in order to identify any discrepancy between medication ordered and patient's list [3]. In addition, patient's understanding of medications and communicating medication reconciliation to other healthcare providers are also an integral part of medication reconciliation. Systemic review of 26 studies done in 2012 revealed significant decrease in medication errors and subsequent decrease in adverse drug reactions [4].

Unfortunately, medication reconciliation is rigged with human errors. These errors lead to unintended discrepancies in medications prescribed for the patient, which usually occur during admission, discharge or outpatient follow-up. Most of these errors are done at the time of discharge with one study indicating $70.7 \%$ patients having at least one actual or potential unintentional discrepancy [5]. Intra hospital transfers are second to hospital discharge in medication discrepancies with $62 \%$ of patients having at least one medication error [6]. 
However, hospital admission is associated with medication errors in $53.6 \%$ of patients [7]. In $30 \%$ of cases, these medical discrepancies can lead to potential harm of patients causing 1.5 million preventable adverse drug events annually; and putting an economic burden of $\$ 3$ billion per year on healthcare $[8,9]$. One of the important causes of such errors is poor communication and loss of information during the transition of care causing approximately half of all medication errors and $20 \%$ of all adverse drug events $[10,11]$.

Medical reconciliation requires collaboration between healthcare providers, nurses and pharmacists. In various studies, pharmacist involvement in medication reconciliation showed decreased medication errors and improved patient outcome $[12,13]$. Another study revealed nurse-pharmacy communication can significantly reduce medication errors [14]. Similarly, interdisciplinary approach between hospital, nursing home and pharmacy in the implementation of medical reconciliation has been shown to improve outcome especially those with chronic conditions [15]. Patient centered approach involving active communication with patients and ensuring effective participation in medical reconciliation can decrease medication errors [16]. The benefits of medication reconciliation extend beyond hospital setting when it can be equally effective in ambulatory setting [17].

Our study demonstrates how small and simple interventions like educating the residents, nurses, physician and patients about the importance of accurate and comprehensive medical reconciliation can effectively improve the number of unintended discrepancies. Through our study we were able to quantify both the accuracy of our existing medication reconciliation process - specifically the patient discharge medication list with outpatient follow-up and measure the change in same after deployment of internally developed interventions designed to increase clinician and patient awareness and improve medication reconciliation accuracy; with the anticipation that in so doing would subsequently enhance patient safety and care.

Kripalani et al have demonstrated the most common types of discrepancies were omission of a medication (34.5\%), incorrect dose (32.9\%) or frequency (15.9\%), or an additional medication that should not have been on the list $(11.9 \%)$. Forms of non adherence included missed doses (48.3\%), premature discontinuation of a medication (18.0\%), failure to fill (10.0\%) or delays in filling (4.7\%) a prescription, taking a medication less often $(9.0 \%)$ or more often $(2.4 \%)$ than prescribed, and taking smaller (4.3\%) or larger (2.4\%) doses than prescribed. Discrepancy of omission was considered to be of significant severity to lead to adverse drug events while discrepancy in dose was considered to of serious severity [18]. Our study demonstrates that simple interventions could decrease discrepancies of omission and dosage substantially to prevent adverse drug events.

In a retrospective analysis, medication reconciliation was conducted over a time frame of six months. A total of 233 medication discrepancies were identified. The medications enlisted in the electronic medical records were verified by patient interviews. The authors concluded that putting an end date to a prescribed medication could minimize errors [18].
In a prospective randomized study Hawes et al. reported a decrease in 30-day readmission and emergency department visits after a pharmacy driven clinic visit and intervention. ( $0 \%$ vs. $40.5 \%$, p <0.001). The intervention entailed a clinical visit to the pharmacist and addressing each medication, removing the discrepancies and creation of "Best Possible Medication Discharge List" [19]. In a study by Stewart and Lynch conducted on 219 patients the descripencies were idenitifed in $97.5 \%$ of the patients. After medication reconciliation there was a reduction in the discrepancy from $97.5 \%$ to $82.5 \%$. However the mean rate for the discrepancy did not achieve statistical significance (0.994 vs. $0.715 \mathrm{p}=0.255$ ) [20].

Medication reconciliation has its own flaws. Meticulous reconciliation is time consuming and labor intensive for physicians and the medical team. In a busy practice, detailed medication reconciliation may be easily viewed as an insignificant and redundant process with low probability of affecting patient's health [21]. Hence educating residents, nurses and physician to improve patient's knowledge and familiarity with his medication regimen is a major step in strengthening medication reconciliation. Frequent reminders and notification to the patients informing them about their appointment and refills may significantly assist in increasing patient compliance and provide appropriate medical supervision after discharge.

The present study has its own limitations. Firstly, the limited sample size may have affected the power of our study. To address this issue a non-parametric analysis was performed. Secondly, the information obtained from the patient via telephone call was performed mostly by our trained clinic nurses but errors made by patients in reporting drug, duration, or dose, or by nurses cannot be verified. Conducting a prospective randomized trial with blinding and a well-defined control group in a multiinstitutional study may substantiate our findings.

\section{Conclusion}

In conclusion, our intervention improved the accuracy of patient current medication list in the electronic records. In an era where EMR is used for patient care across multiple offices by multiple providers, it is important for primary care physicians to keep medication lists as accurate as possible. For clinics and hospitals, medication reconciliation can enhance delivery of high value cost conscious care to the patients by reducing medication errors.

\section{Competing Interests}

None.

\section{References}

[1] Thomsen LA, Winterstein AG, Søndergaard B, Haugbølle LS, Melander A. Systematic review of the incidence and characteristics of preventable adverse drug events in ambulatory care. Ann Pharmacother. 2007 Sep; 41(9): 1411-26. Epub 2007 Jul 31. 
[2] Boockvar KS, Carlson LaCorte H, Giambanco V, et al. Medication reconciliation for reducing drug-discrepancy adverse events. Am J Geriatr Pharmacother 2006; 4: 236.

[3] Greenwald JL, Halasyamani L, Greene J, et al. Making inpatient medication reconciliation patient centered, clinically relevant and implementable: a consensus statement on key principles and necessary first steps. J Hosp Med 2010; 5:477.

[4] Mueller SK, Sponsler KC, Kripalani S, Schnipper JL. Hospitalbased medication reconciliation practices: a systematic review. Arch Intern Med 2012; 172:1057.

[5] Wong JD, Bajcar JM, Wong GG, et al. Medication reconciliation at hospital discharge: evaluating discrepancies. Ann Pharmacother 2008; 42:1373.

[6] Lee JY, Leblanc K, Fernandes OA, et al. Medication reconciliation during internal hospital transfer and impact of computerized prescriber order entry. Ann Pharmacother 2010; 44: 1887.

[7] Cornish PL, Knowles SR, Marchesano R, et al. Unintended medication discrepancies at the time of hospital admission. Arch Intern Med 2005; 165: 424.

[8] Vira T, Colquhoun M, Etchells EE. Reconcilable differences: correcting medication errors at hospital admission and discharge. Qual Saf Health Care 2006; 15: 122-6.

[9] Institute of Medicine. Preventing Medication Errors. Washington, DC: The National Academies Press; 2007.

[10] Barnsteiner JH. Medication reconciliation: transfer of medication information across settings: keeping it free from error. J Infus Nurs. 2005; 28(2 suppl):31-6.

[11] Rozich J, Roger R. Medication safety: one organization's approach to the challenge. J Clin Outcomes Manag. 2001; 8: 27-34.

[12] Murphy EM, Oxencis CJ, Klauck JA, et al. Medication reconciliation at an academic medical center: implementation of a comprehensive program from admission to discharge. Am J Health Syst Pharm. 2009; 66: 2126-31.

[13] Schnipper JL, Kirwin JL, Cotugno MC, et al. Role of pharmacist counseling in preventing adverse drug events after hospitalization. Arch Intern Med. 2006; 166: 565-71.
[14] Feldman, Costa Et al. Nurse-pharmacist collaboration on medication reconciliation prevents potential harm. Journal of Hospital Medicine. 2012 May-Jun: 7(5): 396-401.

[15] Schuetz B, Mann E, Everett W. Educating health professionals collaboratively for teambased primary care. Health Affairs. 29 No. 8 (2010): 1476-1480.

[16] Gleason KM, McDaniel MR, Feinglass J, Baker DW, Lindquist L, Liss D, et al. Results of the Medications at Transitions and Clinical Handoffs (MATCH) study: an analysis of medication reconciliation errors and risk factors at hospital admission. J Gen Intern Med 2010; 25: 441-7.

[17] Nassaralla CL, Naessens JM, Chaudhry R, Hansen MA, Scheitel $\mathrm{SM}$. Implementation of a medication reconciliation process in an ambulatory internal medicine clinic. Quality \& Safety in Health Care. 2007; 16(2): 90-94.

[18] Kripalani S1, Roumie CL, Dalal AK, Cawthon C, Businger A, Eden SK, Shintani A, Sponsler KC, Harris LJ, Theobald C, Huang RL, Scheurer D, Hunt S, Jacobson TA, Rask KJ, Vaccarino V, Gandhi TK, Bates DW, Williams MV, Schnipper JL; PILL-CVD (Pharmacist Intervention for Low Literacy in Cardiovascular Disease) Study Group. Effect of a pharmacist intervention on clinically important medication errors after hospital discharge: a randomized trial. Ann Intern Med. 2012 Jul 3; 157(1): 1-10.

[19] Orrico KB. Sources and types of discrepancies between electronic medical records and actual outpatient medication use. J Manag Care Pharm. 2008 Sep; 14(7):626-31.

[20] Hawes EM, Maxwell WD, White SF, Mangun J, Lin FC. Impact of an outpatient pharmacist intervention on medication discrepancies and health care resource utilization in posthospitalization care transitions. J Prim Care Community Health. 2014 Jan 1; 5(1): 14-8.

[21] Stewart AL, Lynch KJ. Medication discrepancies despite pharmacist led medication reconciliation: the challenges of maintaining an accurate medication list in primary care. Pharm Pract (Granada). 2014 Jan; 12(1):360. Epub 2014 Mar 24. 